Article

\title{
Analytical Modelling of a Refractive Index Sensor Based on an Intrinsic Micro Fabry-Perot Interferometer
}

\author{
Everardo Vargas-Rodriguez ${ }^{1}$, Ana D. Guzman-Chavez ${ }^{1, *}$, Martin Cano-Contreras ${ }^{1,2}$, \\ Eloisa Gallegos-Arellano ${ }^{1,3}$, Daniel Jauregui-Vazquez ${ }^{4}$, Juan C. Hernández-García ${ }^{4}$, \\ Julian M. Estudillo-Ayala ${ }^{4}$ and Roberto Rojas-Laguna ${ }^{4}$
}

1 Departamento de Estudios Multidisciplinarios, División de Ingenierías, Universidad de Guanajuato, Av. Universidad s/n, Col. Yacatitas, Yuriria, Gto., C.P. 38940, Mexico;

E-Mails: evr@ugto.mx (E.V.-R.); mcano_cco@utsoe.edu.mx (M.C.-C.); egallegos@ugto.mx (E.G.-A.)

2 Departamento de Tecnologías de la Información y Comunicación, Universidad Tecnológica del Suroeste de Guanajuato, Carretera Valle-Huanímaro km. 1.2,

Valle de Santiago, Gto., C.P. 38400, Mexico

3 Departamento de Mecatrónica, Universidad Tecnológica de Salamanca, Av. Universidad Tecnológica \#200, Col. Ciudad Bajío, Salamanca, Gto., C.P. 36766, Mexico

4 Departamento de Electrónica, División de Ingenierías, Universidad de Guanajuato, Carretera Salamanca-Valle de Santiago km 3.5 + 1.8, Comunidad de Palo Blanco, Salamanca, Gto., C.P. 36885, Mexico; E-Mails: jaureguid@ugto.mx (D.J.-V.); jchernandez@ugto.mx (J.C.H.-G.); julian@ugto.mx (J.M.E.-A.); rlaguna@ugto.mx (R.R.-L.)

* Author to whom correspondence should be addressed; E-Mail: ad.guzman@ugto.mx; Tel.: +52-445-4589040 (ext. 1718).

Academic Editor: Gangbing Song

Received: 22 July 2015 / Accepted: 9 October 2015 / Published: 15 October 2015

\begin{abstract}
In this work a refractive index sensor based on a combination of the non-dispersive sensing (NDS) and the Tunable Laser Spectroscopy (TLS) principles is presented. Here, in order to have one reference and one measurement channel a single-beam dual-path configuration is used for implementing the NDS principle. These channels are monitored with a couple of identical optical detectors which are correlated to calculate the overall sensor response, called here the depth of modulation. It is shown that this is useful to minimize drifting errors due to source power variations. Furthermore, a comprehensive analysis of a refractive index sensing setup, based on an intrinsic micro Fabry-Perot Interferometer (FPI) is described. Here, the changes over the FPI pattern as the exit refractive
\end{abstract}


index is varied are analytically modelled by using the characteristic matrix method. Additionally, our simulated results are supported by experimental measurements which are also provided. Finally it is shown that by using this principle a simple refractive index sensor with a resolution in the order of $2.15 \times 10^{-4}$ RIU can be implemented by using a couple of standard and low cost photodetectors.

Keywords: Fabry-Perot interferometer; fiber optics sensors; interferometry; non dispersive sensing; tunable laser spectroscopy

\section{Introduction}

In recent years fiber optic refractive index (RI) sensors have gain attention since these have interesting characteristics which allow them to be used in a broad range of chemical and biological sensing applications [1,2]. Moreover, fiber optic RI sensors can be implemented in different ways, such as by using fiber Bragg gratings [3-6], long period gratings [7-9], interferometers [10-12], resonators [13-16] and tapers $[2,9,17,18]$. Among these the Fabry-Perot interferometer (FPI) is a very popular option since it can be fabricated in different ways [19-35]. For instance, FPIs can be implemented by forming an air cavity between two optical fiber segments placed within a microcapillary [19,20]. Another technique consists in forming an open micro-notch cavity by micromachining either a single mode or a photonic crystal fiber with a fs laser [21,22]. FPIs at the tip of an optical fiber have also been implemented by different fabrication processes [23-29]. As an example, the technique presented by [23] consisting in produce a circular hole at the center of the cross-section area of a single-mode fiber (SMF) by means of a laser micromachining system. Afterwards, this micro-machined segment of SMF is spliced to another segment of optical fiber in order to form an air cavity. Finally the spliced fiber is cleaved forming one of the FPI optical mirrors [23], the other mirror will be the core of the micromachined SMF itself. Another way to form an FPI at the tip of an optical fiber is by fabricating an air microbubble [25-29]. In these FPIs the air cavity is formed by splicing a segment of hollow core photonic crystal fiber (HCPCF) to one SMF segment. Afterwards the HCPCF is cleaved by applying electrical discharges with a fusion splicer. Here a bubble of air is trapped within the HCPCF forming the FPI air microcavity and the HCPCF material that gets fused, when the electrical discharges are applied, will form the exit FPI mirror. Fiber tip FPIs can be used for refractive index sensing since within the exit FPI mirror multiple internal reflections will occur, if it has flat and parallel surfaces [36]. These reflections generate a well defined FPI spectrum, which fringe contrast depends on the refractive index of the medium around the FPI sensor tip. Usually the spectral response of this FPIs is analytically modelled by calculating the reflection coefficients of the three reflecting surfaces [25-30] which have very low reflectivity mirrors. In this paper the spectral response of the microcavity FPI is modelled by using the characteristic matrix method. This analytical model is not limited to solve FPIs formed by low reflectivity mirrors and on the contrary it can be used to spectrally model similar fiber FPIs tips that can have longer optical thickness and mirrors with higher refractive indexes [33-35]. Moreover, in some cases the optical thickness of the mirrors and the numerical aperture of the fiber can induce effects on the spectral FPI fringe pattern, 
all these effects can be modelled by applying the characteristics matrix method. Furthermore, this mathematical model allowed us to characterize a refractive index sensor.

Besides the interferometric analysis and characterization of the FPI it is necessary to implement a sensor configuration that allows us to determine in a simple way the refractive index measurements. Therefore in this work it is proposed to implement a sensor setup based on a combination of the Non Dispersive Sensing (NDS) and the Tunable Laser Spectroscopy (TLS) principles. Here in order to have one reference and one measurement channels a single-beam dual-path configuration is used for implementing the NDS principle. These channels are monitored with a couple of identical optical detectors, which output signals are correlated to calculate the overall sensor response, called here the depth of modulation. It is shown that this is useful to minimize drifting errors due to source power variations. By using this configuration it is shown that a sensor with a sensitivity of $3.25 \mathrm{~V} / \mathrm{RIU}$ and a resolution of $2.15 \times 10^{-4} \mathrm{RIU}$, within the refractive index range from 1.3 to 1.7 , can be implemented. Finally, experimental measurements for the FPI characterization and for the overall sensor response are provided to support our simulation results.

\section{Refractive Index Sensing Setup}

In this work a refractive index sensing setup based on a micro-FPI and on the non-dispersive sensing (NDS) principle of operation is presented. In general sensors based on NDS cannot resolve the spectrum of the sample, since all wavelengths provided by the source reach the detector at the same time and therefore it is neither possible to determine at which frequency a change in the spectrum occurs nor its intensity. However, these sensors can be implemented relatively easy, for instance the simplest NDS setup consists in passing a light beam through the analyte and the transmitted or reflected light is measured by an optical detector. Here the changes occurring in the analyte will induce a power variation which is observed by the detector. However due to the simplicity of this configuration it can be very sensitive to variations in the light source, inducing measurement errors. Therefore, there are several designs based on the NDS principle, but some of these configurations are designed to increase the sensitivity, the resolution and their ability to "identify" changes that are due only to the analyte and discard or compensate some other external changes, for instance variations in the light source. In the literature very different arrangements for different applications can be found, based on the NDS principle [37-40]. One possible way to classify these sensors is by the number of sources (beams), wavelength (filters), paths and detectors (channels) that they use. Some of the basic and simplest possible configurations are: single-beam single-wavelength [39], dual-beam single-wavelength [38], single-beam dual-wavelength [37], single-beam dual-path [40] and tunable laser spectroscopy (TLS) [41,42]. In our case we propose a refractive index sensor based on the NDS principle in which the single-beam dual-path and the TLS configurations are combined (Figure 1).

For this configuration only one laser source was used, which is divided into two paths by a 50/50 fiber coupler. One of these paths will be our reference channel and the second one will be the measurement channel, which satisfies the single-beam and dual-path concept. Moreover, the source is a laser emitting at $1550 \mathrm{~nm}$ and it can be tuned to a few nanometers, which is the basis of the TLS. Moreover, the beam on the measurement channel travels toward the FPI through the circulator (6015-3, Thorlabsv, Newton, NJ, USA) from the port 1 to the port 2. Afterwards, the reflected power $\left(P_{M E}\right)$ is 
detected by the measurement channel detector, while the reference channel power $\left(P_{R E}\right)$ is monitored by the reference detector. In this sensing arrangement the FPI spectrum is formed by a periodic fringe pattern which contrast will depends on the refractive index of the exit medium. Therefore the measurement channel detector will observe changes depending on the refractive index of the external medium $\left(n_{e}\right)$ while the reference channel detector will observe ideally a constant signal. These monitored signals will be correlated in order to obtain the depth of modulation, which can be useful to reduce drifting errors due to variations on the laser source. Hence in next section we will analyse and characterize the spectral response of the FPI as a function of the exit refractive index and later the overall output of our NDS arrangement.

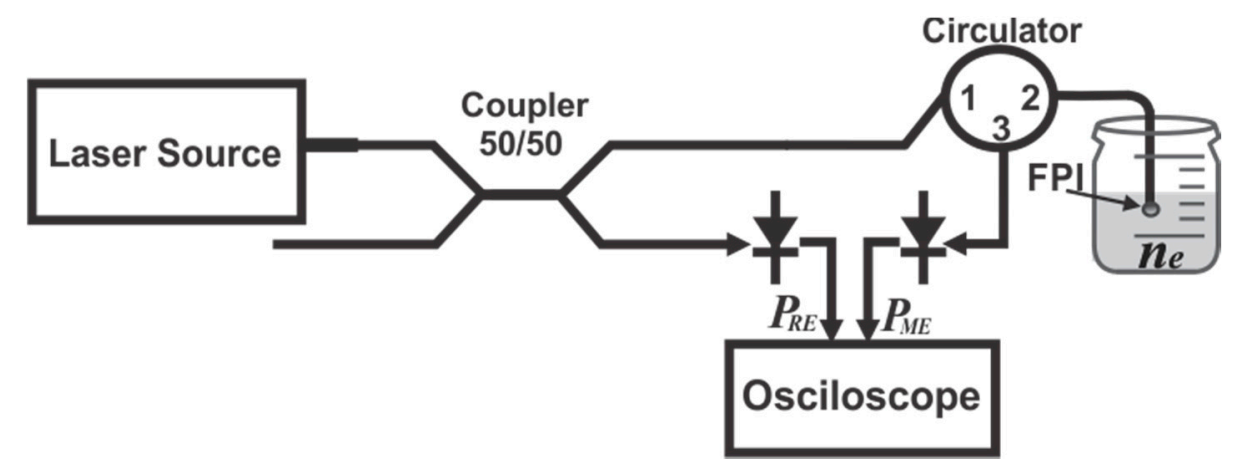

Figure 1. Refractive index sensing setup.

\section{Characterization of the Micro FPI}

In order to characterize the spectral response of the FPI as a function of the exit refractive index medium the setup shown in Figure 2a was implemented. Here, the light of a pigtailed diode laser emitting at $\lambda=980 \mathrm{~nm}$, delivering a maximum output power of $200 \mathrm{~mW}$, was coupled to a wavelength division multiplexer (QFBGLD-980-200, Qphotonics, Ann Arbor, MI, USA) to pump an erbium doped fiber (F-EDF-T3, Newport, Newport Corporation, Irvine, CA, USA) of $3.4 \mathrm{~m}$ length. Afterwards, the luminescence generated by the EDF travels toward the FPI through the circulator (Thorlabs 6015-3) from the port 1 to the port 2. Finally, the reflected interference spectrum of the FPI was monitored at the port 3 of the circulator by using an optical spectrum analyser (OSA, AQ6370C, Yokogawa, Musashino-shi, Tokyo, Japan) with a resolution of $0.02 \mathrm{~nm}$. In order to measure refractive index changes, the FPI was placed into a cuvette which was filled with different liquids that have calibrated refractive indexes. In this arrangement the sensor head is the intrinsic FPI which is based on an air microcavity. This was fabricated by splicing a segment of hollow core photonic crystal fiber (HC-1064-19 Cells Fiber Crystal, Blokken, Birkerød, Denmark) to a standard single mode fiber (SMF). Here to splice both fibers a conventional arc fusion splicer (Fitel-S175) was used and the fabrication process described in detail by [28] was followed. In this process after fibres get spliced multiple electric discharges are applied in order to cleave the photonic crystal fiber. After this step an air microcavity FPI is obtained at the tip of the SMF. A picture of the fabricated MFPI is shown in Figure 2b. 


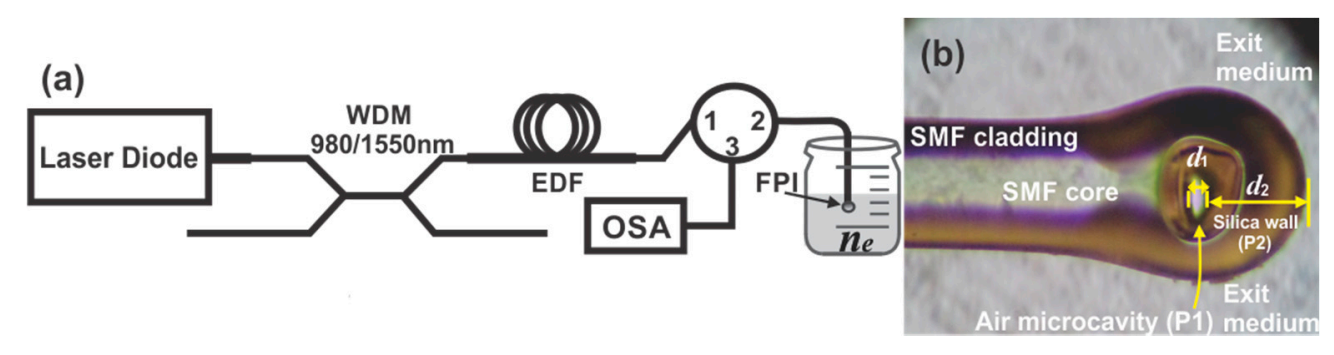

Figure 2. (a) Setup used to characterize the micro FPI spectral response as a function of the exit medium refractive index; (b) Picture of the fabricated FPI.

\section{FPI Principle of Operation}

The sensor head is basically a simple FPI which has the structure shown in Figure 3. Here the FPI mirrors are formed by the SMF core and by the thin silica wall at the tip of the fiber, which are separated by the air microcavity (Figure 3). In order to determine the overall reflectivity spectrum of the FPI structure we considered it as a pile of plane and parallel plates. We assumed this because our structure is a near planar FPI since the air microcavity length is much shorter than the radius of curvature of the exit mirror $\left(d_{1}<<r\right)$ (Figure 2b) [43-46]. For the case of near planar FPIs the finesse and the maximum transmission will be affected due to parabolic errors induced by mirrors curvature $[45,46]$. These mirror parallelism errors will strongly affect the FPI spectrum particularly for high reflectivity values [45]. Hence as we have a near planar FPI with very low reflectance mirror $(\approx 0.04)$ therefore can be expected that the parabolic errors will not strongly affect the FPI spectral response. Consequently the structure can be assumed as a simple FPI formed by two flat plates. The first plate (P1) is the air microcavity with length $d_{1}$, while the second plate (P2) is the thin silica wall at the tip of the FPI with thickness $d_{2}$. Finally the incident medium of the pile of plates is the SMF core, with a refractive index $n_{0}$, and the exit medium, which is surrounding the FPI tip, has a refractive index $n_{e}$.

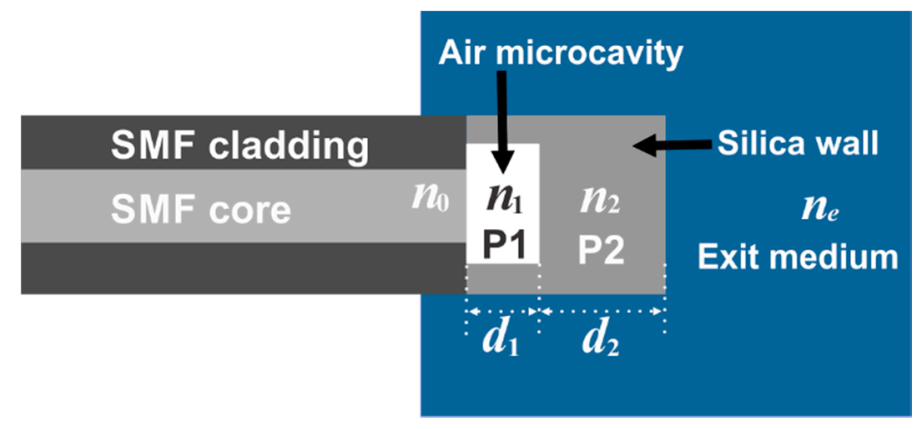

Figure 3. FPI structure.

Hence, as the overall FPI is seen as a pile of plates therefore the final FPI reflectivity fringe pattern can be calculated by using the characteristic matrix. By using this method all reflections occurring at each interface of the FPI are taken into account which allow us to simulate with higher precision the reflectivity spectrum. The characteristic matrix of the whole medium can be expressed as [47]:

$$
\mathbf{M}=\prod_{j=1}^{N} \mathbf{M}_{j}=\left[\begin{array}{ll}
a & i b \\
i c & d
\end{array}\right]
$$


where $\mathbf{M}_{j}$ is the characteristic matrix of the $j$-th plate, which for an unpolarised beam is given by [48]:

$$
\mathbf{M}_{j}=\left[\begin{array}{cc}
\cos \left(\frac{2 \pi n_{j} d_{j} \cos \theta_{j}}{\lambda}\right) & -\frac{i}{n_{j}} \sin \left(\frac{2 \pi n_{j} d_{j} \cos \theta_{j}}{\lambda}\right) \\
-i n_{j} \sin \left(\frac{2 \pi n_{j} d_{j} \cos \theta_{j}}{\lambda}\right) & \cos \left(\frac{2 \pi n_{j} d_{j} \cos \theta_{j}}{\lambda}\right)
\end{array}\right]
$$

where $n_{j}, d_{j}, \theta_{j}$ are the refractive index, the thickness, and the incident angle of the $j$-th plate respectively and $\lambda$ is the wavelength. The reflectance of this plate assembly can be obtained by using the following definition [48]:

$$
\left[\begin{array}{l}
B \\
C
\end{array}\right]=\left[\begin{array}{cc}
a & i b \\
i c & d
\end{array}\right]\left[\begin{array}{l}
1 \\
n_{e}
\end{array}\right]
$$

Thus, the reflection coefficient is given by:

$$
r=\frac{n_{0} B-C}{n_{0} B+C}
$$

and finally the reflectivity of the overall assembly is given by:

$$
R_{F P}\left(\lambda, n_{e}\right)=\left(\frac{n_{0} B-C}{n_{0} B+C}\right)\left(\frac{n_{0} B-C}{n_{0} B+C}\right)^{*}
$$

Therefore in our system the refractive index $n_{0}=1.44$, the P1 has a $n_{1}=1$ and P2 has a $n_{2}=1.44$. Finally the exit medium $n_{e}$ will depend on the material surrounding the micro FPI structure (Figure 2). Simulated reflectivity spectra of the FPI for different $n_{e}$ are shown in Figure 4a. For these simulations we considered $d_{1}=12.7 \mu \mathrm{m}$ and $d_{2}=110.9 \mu \mathrm{m}$. As it can be appreciated in this type of FPI neither the fringe positions nor the free spectral range will change as $n_{e}$ is varied [49-51]. Here only the contrast and the finesse of the spectral fringes occurring by multiple internal reflections within $\mathrm{P} 2$ will be modified (Figure 4b).
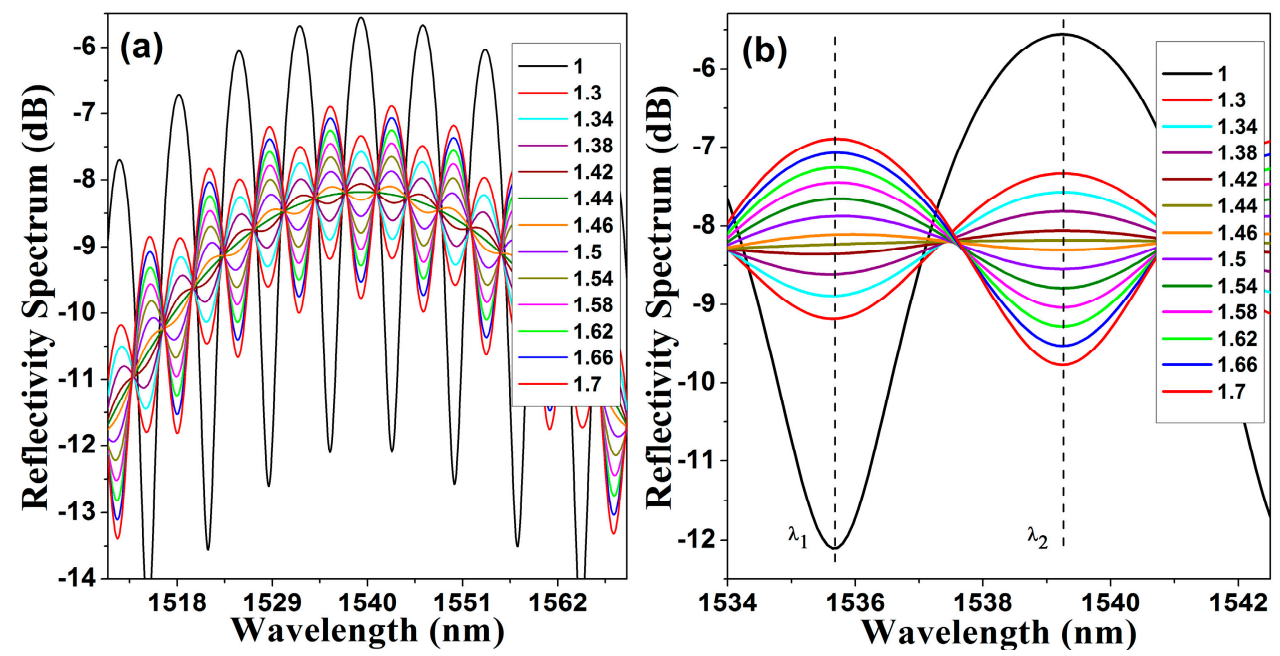

Figure 4. (a) Simulated FPI reflectivity spectra considering an exit medium with different refractive indexes; (b) Detail of the FPI reflectivity spectra showing a single fringe peak and valley. 


\section{Experimental Results of the FPI Spectrum}

In Figure $5 S(\lambda)$ is shown, which is the spectral profile of the luminescence generated by the EDF, which illuminates the micro FPI. Moreover, in this figure it is also shown the measured reflection spectral profile $I_{M}\left(\lambda, n_{e}\right)$ recorded with the OSA (Figure 2). From this measured spectral profile it is possible to determine the exact thickness of the thin silica wall (P2), where multiple internal reflections occur. Here it is important to recall that the Free Spectral Range $(F S R)$, which is the separation between two consecutive fringes, is defined as:

$$
F S R=\frac{\lambda^{2}}{2 n_{2} d_{2}}
$$

Therefore if the measured $F S R=7.38 \mathrm{~nm} \mathrm{~nm}$ then $d_{2}=110.9 \mu \mathrm{m}$, which is very close to the experimentally measured value. The measured air microcavity for this FPI was approximately $d_{1}=12.7 \mu \mathrm{m}$, this was measured by using an optical microscope. Additionally, in Figure 6 it is also shown the simulated spectral profile of the light reflected by the FPI, which is given by $I_{S}\left(\lambda, n_{e}\right)=S(\lambda) R_{F P}\left(\lambda, n_{e}\right)$.

In our FPI insertion losses occurred, which are mainly due to the photonic crystal and single mode fibers splicing. These losses are basically flat over the spectrum (Figure 5) and can be described as $A=I_{M}-I_{S} \approx 13.85 \mathrm{~dB}$. For this calculation we are taking as a reference the case when the exit medium is air $\left(n_{e}=1\right)$.

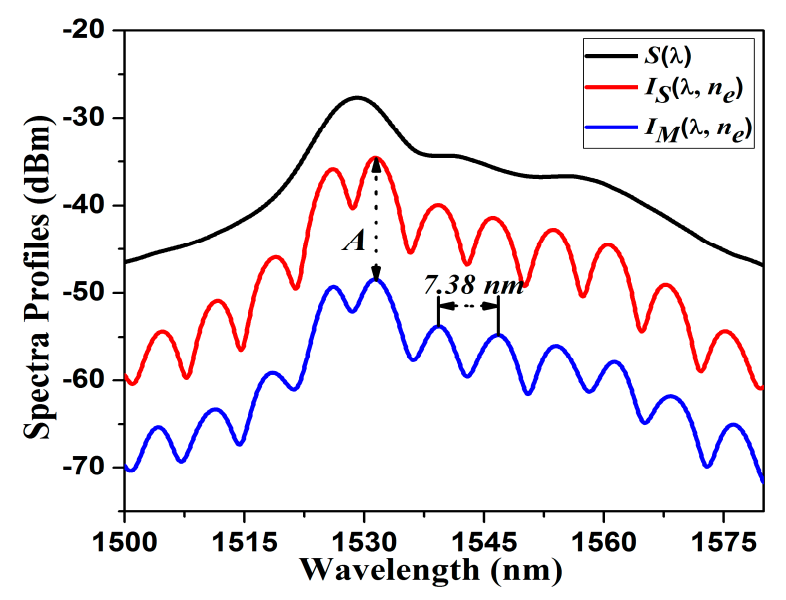

Figure 5. Measured source spectral profile $S(\lambda) ; I_{M}\left(\lambda, n_{e}\right)$ and $I_{S}\left(\lambda, n_{e}\right)$ are the measured and the simulated FPI reflection spectrum respectively. For this case $n_{e}=1$ was considered.

Additionally, the measured FPI reflection spectral profile is shown in Figure 6a. For this experiment the exit medium was changed in order to vary $n_{e}$. In Figure $6 \mathrm{~b}$ a detail of the spectral profile is presented. 

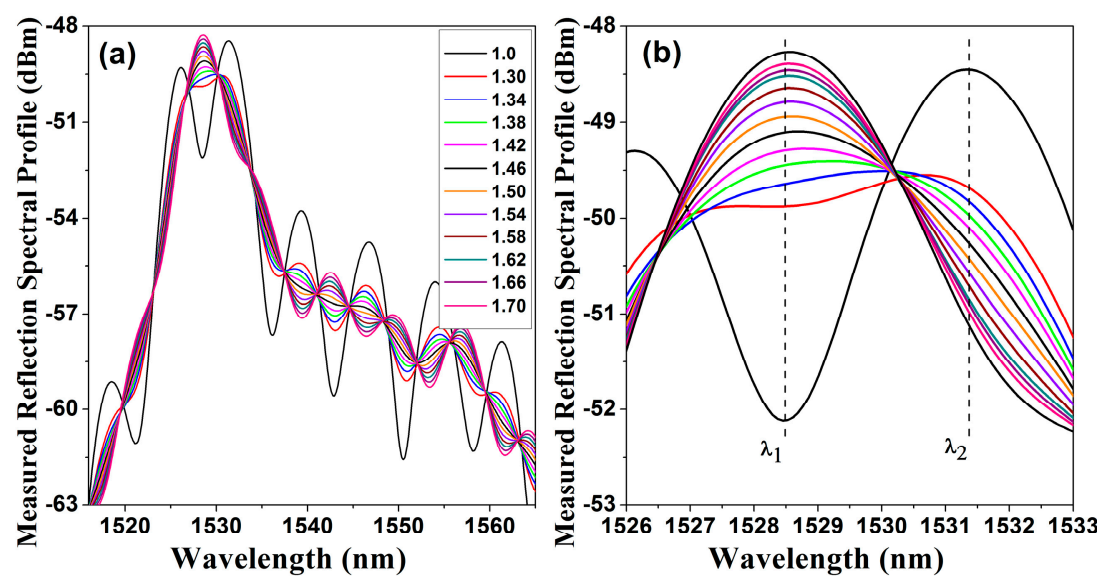

Figure 6. (a) Measured reflected power spectra $I_{M}\left(\lambda, n_{e}\right)$ for different values of exit medium refractive index; (b) detail of the $I_{M}\left(\lambda, n_{e}\right)$ spectra showing only a single spectral FPI fringe.

Characterization of the Amplitude of the FPI Fringes as a Function of the Exit Medium Refractive Index

As the contrast and the finesse of the spectral fringes change as the refractive index of the exit medium is varied, therefore it is possible to detect these changes by measuring the amount of reflected light at certain wavelength. This change will be not linear, since it depends on the reflectivity of the exit interface, formed by mirror (P2) and the exit medium however it is completely predictable. Hence for instance, by using the arrangement shown in Figure 2 the spectral response presented in Figure $6 \mathrm{~b}$ was obtained. From these spectra the fringe that has the maximum reflection level is analysed, in our case this occurs at $\lambda=1528.3 \mathrm{~nm}$. Moreover, the reflected energy, in $\mathrm{dBm}$, as a function of the exit medium refractive index, $I_{M}\left(1528.3, n_{e}\right)$, is presented in Figure 7. For this example, $I_{M}\left(1528.3, n_{e}\right)$ can be fitted very well by a linear function, $I_{F}\left(1528.3, n_{e}\right)$, as follows:

$$
I_{F}\left(1528.3, n_{e}\right)=3.906 n_{e}-54.87 \text {, for } 1.30 \leq n_{e} \leq 1.70
$$

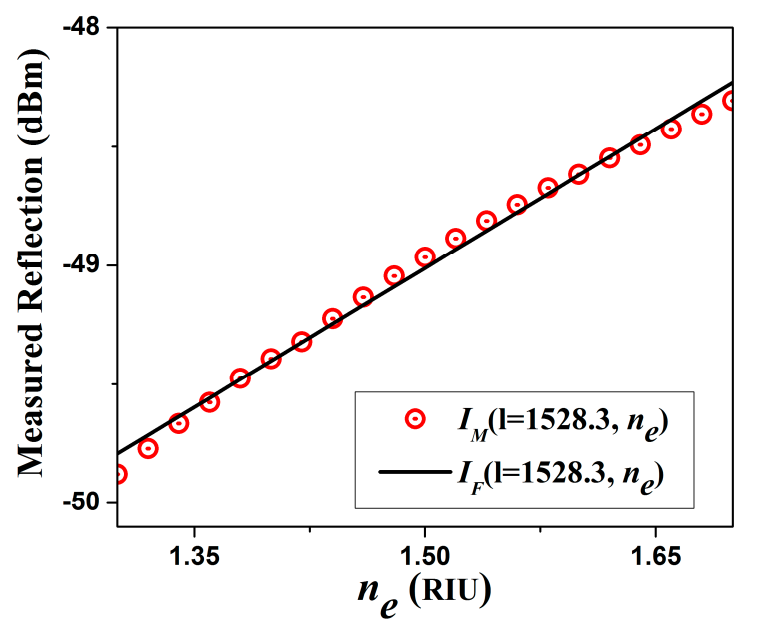

Figure 7. Measured reflected energy at $\lambda=1528.3 \mathrm{~nm}$ as a function of the exit medium refractive index and its linear fitting. 
This change in reflected power can be taken into advantage to implement a refractive index sensor. The main problem is that we will need a very narrow filter at $\lambda=1528.3 \mathrm{~nm}$ in order to implement a practical sensor. Otherwise is to replace the broad band source by a laser that can be tuned near to the peak or valley of one of the FPI fringes. Here we proceed to implement a practical sensor based on a tuneable fiber laser.

\section{Analysis of the Non Dispersive Refractive Index Sensor}

A single-beam single-path ND sensor can be implemented in a relatively simple way, by replacing the broadband source by a single line laser. In general terms the expected sensor response will be described as shown in Figure 6. However this kind of sensor can be sensitive to drifting errors due to different factors such as instability in the laser line position, source power instability and aging of the source and detector. Hence the single-beam dual-path configuration (Figure 1) is useful to reduce this type of error. In this arrangement the energy reaching the measurement detector is directly described by the integrated optical FPI reflectance, which can be expressed as:

$$
P_{M}\left(n_{e}\right)=\int_{\lambda 1}^{\lambda 2} L(\lambda) R\left(\lambda, n_{e}\right) d \lambda
$$

where $L(\lambda)$ is the laser emission profile and $R\left(\lambda, n_{e}\right)$ is the FPI reflectance. The power of the reference channel can be described as:

$$
P_{R}=\int_{\lambda 1}^{\lambda 2} L(\lambda) d \lambda
$$

In order to simulate the energy reaching both detectors $L(\lambda)$ as was assumed as an ideal laser with Gaussian shape, central wavelength at $1550 \mathrm{~nm}$, spectral width of $20 \mathrm{pm}$ and normalized amplitude. The simulated overall output power of the measurement channel $P_{M S}\left(n_{e}\right)$ and of the reference channel output $P_{R S}$ are shown in Figure 8a. For this simulation the scale of the reference and the measurement channels are different since we are considering that both signals are amplified with different gain factors. This is because the measurement channel will have much more losses that the reference channel.

In our experiment a couple of model DET01CFC InGaAs detectors from Thorlabs Inc. were used, which have a noise equivalent power $(N E P)$ of $1.5 \times 10^{-15} \mathrm{~W} / \sqrt{\mathrm{Hz}}$, Responsivity $(G)$ of approximately $1 \mathrm{~A} / \mathrm{W}$, dark current $\left(I_{\text {Dark }}\right)$ of $700 \mathrm{pA} @ 1550 \mathrm{~nm}$ and the minimum load resistance $\left(R_{L}\right)$ allowed is $50 \Omega$. For these detectors the generated photocurrent $\left(I_{P D}\right)$ is defined as the product of the incident power $(P)$ times the responsivity $I_{P D}=P G$. Moreover the output in terms of volts is given as $V O=\left(I_{P D}+I_{\text {Dark }}\right) R_{L}$. Here it is important to recall that the NEP expresses the rms incident radiant power per square root bandwidth necessary to produce a signal to noise ratio of one [52]. The optical detector noise can be generated by different sources, either internal or external. For instance, some internal noise sources are Johnson, shot, $1 / f$, and generation recombination, and some external sources are photon flux, the interface electronics and microphonic noise [52]. Therefore, to have a $S / N=1$ it is necessary that $P=N E P$, this considering that $\Delta f=1$. Consequently the minimum detectable power that can be detected must be equal to $1.5 \times 10^{-15} \mathrm{~W}$, which will produce a minimum output voltage described by:

$$
V o_{\text {min }}=\left(1.5 \times 10^{-15} \mathrm{~W} \times 1 \frac{\mathrm{A}}{\mathrm{W}}+700 \times 10^{-12} \mathrm{~A}\right) R_{L}
$$


In our case we used a $R_{L}=50 \Omega$ for the reference channel and $1 \mathrm{M} \Omega$ for the measurement channel. Hence, for the measurement channel $V o_{\min } \approx 7 \times 10^{-4} \mathrm{~V}$ within $1.3 \leq n_{e} \leq 1.7$. Now, the experimentally measured voltage readings for each channel at different exit refractive index are shown in Figure $8 \mathrm{~b}$. From these measurements it is possible to determine that the sensor has a sensitivity of $3.25 \mathrm{~V} / \mathrm{RIU}$. Consequently, the resolution of the sensor will be in the order of $2.15 \times 10^{-4}$ RIU. From Figure 8 it is clear that the tendency of the measured voltages is in general similar to the predicted by the simulation. However for this sensor if for some reason there is a change in the source characteristics it will induce an error in the final sensor response. For instance the sensor itself is expected to have more tolerance to small variation in the laser central wavelength since the spectral FSR is relatively large and the fringe contrast is very small. However changes in the power source can induce more significant measurement errors. For this reason some authors have proposed to correlate the reference and the measurement channels to calculate the depth of modulation which can useful to reduce this type of error.
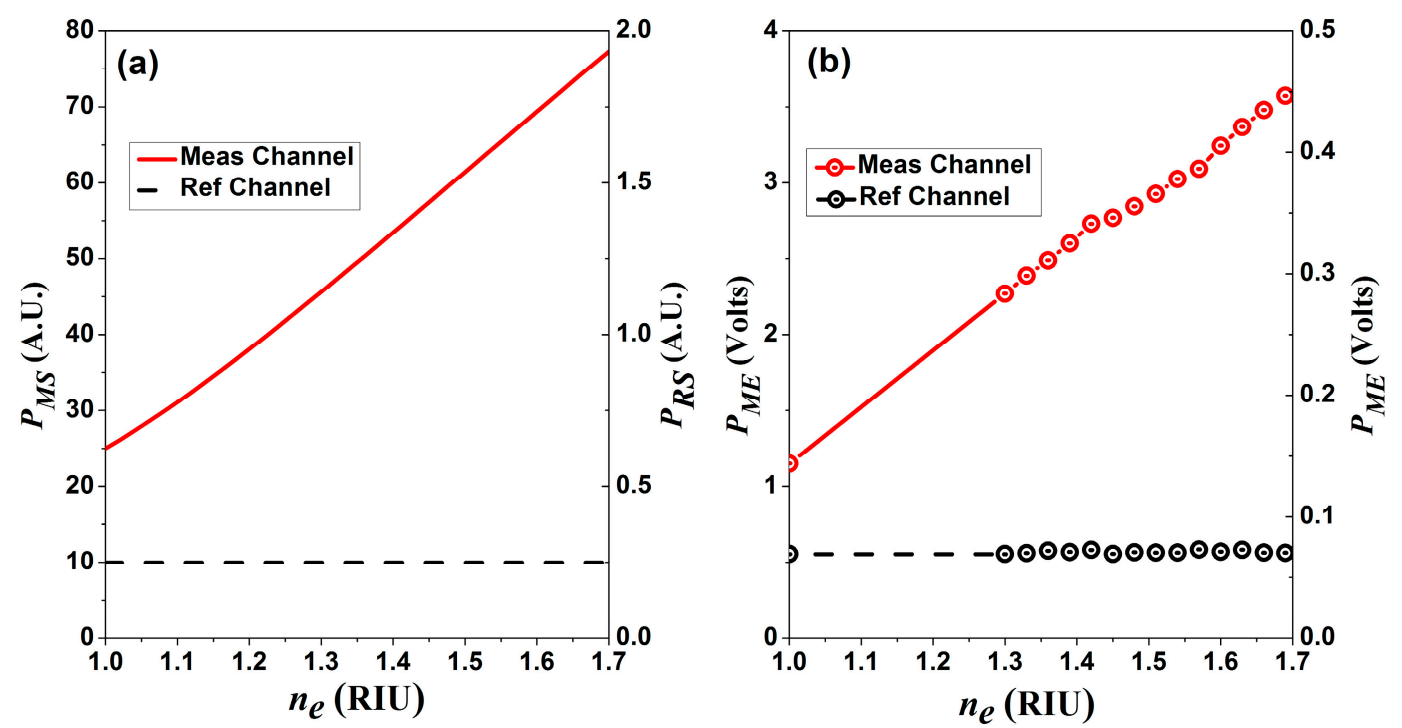

Figure 8. Detector outputs as a function of the exit refractive index, (a) simulated results; (b) experimental measurements.

\section{Depth of Modulation}

In the sensing setup any variation occurring at the source will affect in the same proportion both channels, therefore by correlating both signals it is possible to minimize effects in the sensor response. Therefore instead to measure the sensor response directly from the measurement detector, we prefer to calculate the depth of modulation $(D M)$ [40]. This is defined as the difference between the detected channels signals, divided by the mean of both signals [38] and can be written as:

$$
D M\left(n_{e}\right)=2 \frac{P_{M}\left(n_{e}\right)-k P_{R}}{P_{M}\left(n_{e}\right)+k P_{R}}
$$

In this case $k$ is a proportionality constant to take into account the beam attenuation in the measurement channel, which mainly occur due to the FPI as was previously explained. In this way the $D M\left(n_{e}\right)$ gets normalized and unitless. The proportionality constant for our simulated signals (Figure 9a) 
is given as $k_{S}=P_{M S}\left(n_{e}=1.0\right) / P_{R S}$. Moreover for the measured channel the proportionality constant was determined as $k_{E}=P_{M E}\left(n_{e}=1.0\right) / P_{R E}$. In this way the depth of modulation calculated from simulated and from experimental measurements are presented in Figure 9. Here can be appreciated that the depth of modulation from experimental measurements fits well with our simulation. Now considering that we can detect changes in the RIU with a resolution of $2.15 \times 10^{-4}$ therefore the valid change in the depth of modulation will be of approximately $\Delta D_{m}=1.94 \times 10^{-4}$. The main advantage of this sensing setup is that it compensate variations in the source. Moreover, the depth of modulation calculation can be performed rapidly by a very simple electronic stage based on a low cost microcontroller. Also within this electronic stage can be programmed the equation to convert the depth of modulation into RIU and display the final result on a screen.

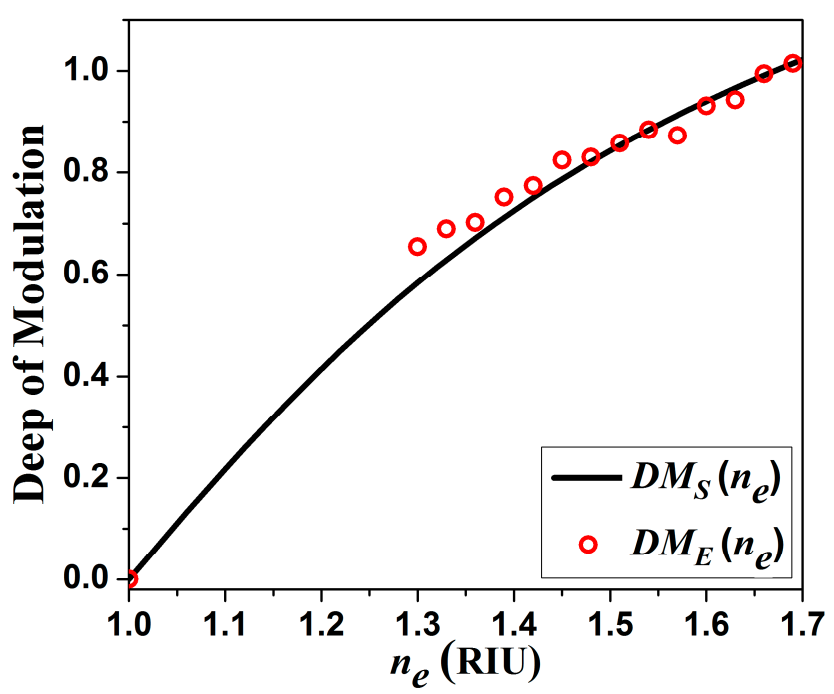

Figure 9. Calculated depth of modulation from simulated and measured signals.

\section{Conclusions}

In this work a comprehensive analysis and characterization of a non-dispersive refractive index sensor, based on an intrinsic FPI was presented. Here the FPI characterization was carried out by considering it as pile of plates and therefore its spectral response is modelled by the characteristic matrix method. Moreover it was shown that in this FPI, multiple internal reflections occur within the exit plate (P2). This effect allows the generation of a FPI fringe pattern, which is sensitive to changes on the refractive index of the exit medium. Due to the changes in the refractive index the fringe pattern contrast and finesse are affected. Therefore to calculate the fringe contrast of spectrum generated at the exit plate (P2) it was considered as a single unbalanced FPI. Here by using the proposed model the contrast and the overall reflection spectrum can be easily calculated. The overall sensor is based on the nondispersive principle with a single-beam dual-path configuration combined with the tunable laser spectroscopy technique. Here by using standard and low cost detectors the resolution achieved is on the order of $2.15 \times 10^{-4}$ RIU. The provided simulation results agree very well with the results obtained experimentally. 


\section{Acknowledgments}

J.C. Hernández-García is supported by CONACYT through chairs for young researchers, project 3155. This work was developed with support in part from FOMIX CONACYT-Gobierno del Estado de Guanajuato under project GTO-2012-C04-193904, in part by CONCYTEG under projects 14-PNPC-DPP-Q182-49 and 14-PNPC-DPP-Q182-47 and in part by PROFECIES 2015.

\section{Author Contributions}

E.V.-R., A.D.G.-C. and R.R.-L. organized and supervised the design, implementation, and verification of the complete sensing system. D.J.-V. and M.J.E.A. fabricated the micro Fabry-Perot interferometer. E.V.-R. and A.D.G.-C. designed the sensor and contributed to the simulation of the sensor. M.C.-C., E.G.-A. and J.C.H.-G. contributed with the implementation of the sensor arrangement and performed the experimental measurements.

\section{Conflicts of Interest}

The authors declare no conflict of interests.

\section{References}

1. Jorge, P.A.S.; Silva, S.O.; Gouveia, C.; Tafulo, P.; Coelho, L.; Caldas, P.; Viegas, D.; Rego, G.; Baptista, J.M.; Santos, J.L.; et al. Fiber optic-based refractive index sensing at inesc porto. Sensors 2012, doi:10.3390/s120608371.

2. Kieu, K.Q.; Mansuripur, M. Biconical fiber taper sensors. IEEE Photonics Technol. Lett. 2006, doi:10.1109/LPT.2006.884742.

3. Liang, W.; Huang, Y.; Xu, Y.; Lee, R.K.; Yariv, A. Highly sensitive fiber bragg grating refractive index sensors. Appl. Phys. Lett. 2005, doi:10.1063/1.1904716.

4. Shao, M.; Qiao, X.; Jiasurname, Z.; Fusurname, H.; Liu, Y.; Li, H.; Zhao, X. Refractive index measurement based on fiber bragg grating connected with a multimode fiber core. Opt. Commun. 2015, doi:10.1016/j.optcom.2015.04.028.

5. Meng, H.; Shen, W.; Zhang, G.; Tan, C.; Huang, X. Fiber bragg grating-based fiber sensor for simultaneous measurement of refractive index and temperature. Sens. Actuators B Chem. 2010, doi:10.1016/j.snb.2010.07.010.

6. Iadicicco, A.; Cusano, A.; Campopiano, S.; Cutolo, A.; Giordano, M. Thinned fiber bragg gratings as refractive index sensors. IEEE Sens. J. 2005, doi:10.1109/JSEN.2005.859288.

7. Guan, C.; Tian, X.; Li, S.; Zhong, X.; Shi, J.; Yuan, L. Long period fiber grating and high sensitivity refractive index sensor based on hollow eccentric optical fiber. Sens. Actuators B Chem. 2013, doi:10.1016/j.snb.2013.07.086.

8. Kapoor, A.; Sharma, E.K. Long period grating refractive-index sensor: Optimal design for single wavelength interrogation. Appl. Opt. 2009, doi:10.1364/AO.48.000G88.

9. Allsop, T.; Floreani, F.; Jedrzejewski, K.; Marques, P.; Romero, R.; Webb, D.; Bennion, I. Refractive index sensing with long-period grating fabricated in biconical tapered fibre. Electron. Lett. 2005, doi:10.1049/el:20058352. 
10. Dianov, E.M.; Vasiliev, S.A.; Kurkov, A.S.; Medvedkov, O.I.; Protopopov, V.N. In-fiber mach-zehnder interferometer based on a pair of long-period gratings. In Proceedings of the 22nd European Conference on Optical Communication, ECOC'96, Oslo, Norway, 19 September 1996; Volume 1, pp. 65-68.

11. Allsop, T.; Reeves, R.; Webb, D.J.; Bennion, I.; Neal, R. A high sensitivity refractometer based upon a long period grating mach-zehnder interferometer. Rev. Sci. Instrum. 2002, doi:10.1063/1.1459093.

12. Pieter, L.S. Long-period grating michelson refractometric sensor. Meas. Sci. Technol. 2004, 15, 1576-1580.

13. Shi, L.; Xu, Y.; Tan, W.; Chen, X. Simulation of optical microfiber loop resonators for ambient refractive index sensing. Sensors 2007, 7, 689-696.

14. Lim, K.-S.; Aryanfar, I.; Chong, W.-Y.; Cheong, Y.-K.; Harun, S.W.; Ahmad, H. Integrated microfibre device for refractive index and temperature sensing. Sensors 2012, 12, 11782-11789.

15. Yang, H.J.; Wang, J.; Wang, S.S. Numerical calculation of temperature sensing in seawater based on microfibre resonator by intensity-variation scheme. J. Eur. Opt. Soc. Rapid Publ. 2014, doi:10.2971/jeos.2014.14047.

16. Sumetsky, M.; Dulashko, Y.; Fini, J.M.; Hale, A.; DiGiovanni, D.J. The microfiber loop resonator: Theory, experiment, and application. Lightwave Technol. J. 2006, doi:10.1109/JLT.2005.861127.

17. Kumar, A.; Subrahmanyam, T.V.B.; Sharma, A.D.; Thyagarajan, K.; Pal, B.P.; Goyal, I.C. Novel refractometer using a tapered optical fibre. Electron. Lett. 1984, doi:10.1049/el:19840370.

18. Monzón-Hernández, D.; Villatoro, J.; Luna-Moreno, D. Miniature optical fiber refractometer using cladded multimode tapered fiber tips. Sens. Actuators B Chem. 2005, doi:10.1016/ j.snb.2005.01.011.

19. Kersey, A.D.; Jackson, D.A.; Corke, M. A simple fibre Fabry-Perot sensor. Opt. Commun. 1983, doi:10.1016/0030-4018(83)90047-0.

20. Vikram, B.; Kent, A.M.; Richard, O.C.; Mark, E.J.; Jennifer, L.G.; Tuan, A.T.; Greene, J.A. Optical fibre based absolute extrinsic Fabry-Pérot interferometric sensing system. Meas. Sci. Technol. 1996, 7, 58-61.

21. Rao, Y.-.J.; Deng, M.; Duan, D.-W.; Yang, X.-C.; Zhu, T.; Cheng, G.-H. Micro Fabry-Perot interferometers in silica fibers machined by femtosecond laser. Opt. Express 2007, doi:10.1364/ OE.15.014123.

22. Wei, T.; Han, Y.; Li, Y.; Tsai, H.-L.; Xiao, H. Temperature-insensitive miniaturized fiber inline Fabry-Perot interferometer for highly sensitive refractive index measurement. Opt. Express 2008, doi:10.1364/OE.16.005764.

23. Ran, Z.L.; Rao, Y.J.; Liu, W.J.; Liao, X.; Chiang, K.S. Laser-micromachined Fabry-Perot optical fiber tip sensor for high-resolution temperature-independent measurement of refractive index. Opt. Express 2008, doi:10.1364/OE.16.002252.

24. Liao, C.R.; Hu, T.Y.; Wang, D.N. Optical fiber Fabry-Perot interferometer cavity fabricated by femtosecond laser micromachining and fusion splicing for refractive index sensing. Opt. Express 2012, doi:10.1364/OE.20.022813.

25. Wang, T.; Wang, M. Fabry-Pérot fiber sensor for simultaneous measurement of refractive index and temperature based on an in-fiber ellipsoidal cavity. IEEE Photonics Technol. Lett. 2012, doi:10.1109/LPT.2012.2212184. 
26. Ma, J.; Ju, J.; Jin, L.; Jin, W.; Wang, D. Fiber-tip micro-cavity for temperature and transverse load sensing. Opt. Express 2011, doi:10.1364/OE.19.012418.

27. Duan, D.-W.; Rao, Y.-J.; Hou, Y.-S.; Zhu, T. Microbubble based fiber-optic Fabry-Perot interferometer formed by fusion splicing single-mode fibers for strain measurement. Appl. Opt. 2012, doi:10.1364/AO.51.001033.

28. Jauregui-Vazquez, D.; Estudillo-Ayala, J.M.; Rojas-Laguna, R.; Vargas-Rodriguez, E.; Sierra-Hernandez, J.M.; Hernandez-Garcia, J.C.; Mata-Chávez, R.I. An all fiber intrinsic Fabry-Perot interferometer based on an air-microcavity. Sensors 2013, 13, 6355-6364.

29. Jiang, X.; Chen, D. Low-cost fiber-tip Fabry-Perot interferometer and its application for transverse load sensing. Prog. Electromagn. Res. Lett. 2014, 48, 103-108.

30. Rao, Y.-J.; Deng, M.; Zhu, T.; Li, H. In-line Fabry-Perot etalons based on hollow-corephotonic bandgap fibers for high-temperature applications. Lightwave Technol. J. 2009, 27, 4360-4365.

31. Rao,Y.-J. Recent progress in fiber-optic extrinsic Fabry-Perot interferometric sensors. Opt. Fiber Technol. 2006, doi:10.1016/j.yofte.2006.03.004.

32. Choi, H.Y.; Park, K.S.; Park, S.J.; Paek, U.-C.; Lee, B.H.; Choi, E.S. Miniature fiber-optic high temperature sensor based on a hybrid structured Fabry-Perot interferometer. Opt. Lett. 2008, doi:10.1364/OL.33.002455.

33. Wang, J.; Dong, B.; Lally, E.; Gong, J.; Han, M.; Wang, A. Multiplexed high temperature sensing with sapphire fiber air gap-based extrinsic Fabry-Perot interferometers. Opt. Lett. 2010, doi:10.1364/OL.35.000619.

34. Li, Y.; Yan, G.; Zhang, L.; He, S. Microfluidic flowmeter based on micro "hot-wire" sandwiched Fabry-Perot interferometer. Opt. Express 2015, doi:10.1364/OE.23.009483.

35. Hou, W.L.; Liu, G.G.; Han, M. A novel, high-resolution, high-speed fiber-optic temperature sensor for oceanographic applications. In Proceedings of the 2015 IEEE/OES Eleventh Current, Waves and Turbulence Measurement (CWTM), St. Petersburg, FL, USA, 2-6 March 2015.

36. Vargas-Rodriguez, E.; Rutt, H.N.; Rojas-Laguna, R.; Alvarado-Mendez, E. Calibration of a gas sensor based on cross-correlation spectroscopy. J. Opt. A Pure Appl. Opt. 2008, 10, doi:10.1088/1464-4258/10/10/104018.

37. Carlson, H. Carbocap - New carbon dioxide sensor electrically tuneable interferometer for infrared gas analysis. Sens. Rev. 1997, 17, 304-306.

38. Dakin, J.P.; Gunning, M.J.; Chambers, P.; Xin, Z.J. Detection of gases by correlation spectroscopy. Sens. Actuators B Chem. 2003, 90, 124-131.

39. Zhang, G.; Lui, J.; Yuan, M. Novel carbon dioxide gas sensor based on infrared absorption. Opt. Eng. 2000, 39, 2235-2240.

40. Goody, R. Cross-correlating spectrometer. J. Opt. Soc. Am. B 1968, 58, 900-908.

41. Menzel, L.; Kosterev, A.A.; Curl, R.F.; Tittel, F.K.; Gmachl, C.; Capasso, F.; Sivco, D.L.; Baillargeon, J.N.; Hutchinson, A.L.; Cho, A.Y.; et al. Spectroscopic detection of biological no with a quantum cascade laser. Appl. Phys. B Lasers Opt. 2001, 72, 859-863.

42. Roller, C.; Namjou, K.; Jeffers, J.D.; Camp, M.; Mock, A.; McCann, P.J.; Grego, J. Nitric oxide breath testing by tunable-diode laser absorption spectroscopy: Application in monitoring respiratory inflammation. Appl. Opt. 2002, 41, 6018-6029. 
43. Vaĭnshteĭn, L.A. Open resonators with spherical mirrors. Sov. Phys. J. Exp. Theor. Phys. 1964, 18, 471-479.

44. Kerner, K.; Rochester, S.M.; Yashchuk, V.V.; Budker, D. Variable free spectral range spherical mirror fabry-perot interferometer. 2003. arXiv:physics/0306144. arXiv.org-Print archive. Available online: http://arxiv.org/abs/physics/0306144 (accessed on 18 June 2003).

45. Ramsay, J.V. Aberrations of Fabry-Perot interferometers when used as filters. Appl. Opt. 1969, 8, 569-574.

46. Hernandez, G. Analytical description of a Fabry-Perot photoelectric spectrometer. Appl. Opt. 1966, $5,1745-1748$.

47. Born, M.; Wolf, E. Principles of Optics; Pergamon Press: Oxford, UK, 1970.

48. Macleod, H.A. Thin-Films Optical Filters; Institute of Physics Publishing: Bristol, UK, 2001.

49. Kuhn, H. New techniques in optical interferometry. Rep. Prog. Phys. 1951, 14, 64-94.

50. Vargas-Rodríguez, E.; Rutt, H.N. An analytical method to find the optimal parameters for gas detectors based on correlation spectroscopy using a Fabry-Perot interferometer. Appl. Opt. 2007, $46,4625-4632$.

51. Vargas-Rodríguez, E.; Rutt, H.; Claudio-Gonzalez, D.; Rojas Laguna, R.; Andrade-Lucio, J.A. Analytical approaches to optimization of gas detection using Fabry-Perot interferometer. In Chemical Sensors: Simulation and Modeling vol. 4: Optical Sensors; Momentum Press: New York, NY, USA, 2013.

52. Dereniak, E.L.; Boreman, G.D. Infrared Detectors and Systems; John Wiley and Sons Inc.: Hoboken, NJ, USA, 1996.

(C) 2015 by the authors; licensee MDPI, Basel, Switzerland. This article is an open access article distributed under the terms and conditions of the Creative Commons Attribution license (http://creativecommons.org/licenses/by/4.0/). 